\title{
Avulsion du listel marginal antérieur du rachis cervical
}

\section{Avulsion of the Cervical Spinal Ring Apophyses}

\section{H. Slimani · P. Kauffmann • P. Le Borgne}

Reçu le 24 janvier 2015; accepté le 3 avril 2015

(C) SFMU et Lavoisier SAS 2015

Un adolescent de 17 ans s'est présenté aux urgences suite à un accident de la voie publique à faible cinétique, responsable d'un traumatisme cervical en hyperextension. Le patient n'a aucun antécédent notable. Outre la cervicalgie post-traumatique, l'examen clinique initial retrouve une rectitude du rachis et une contracture douloureuse des masses paravertébrales. La mobilisation prudente rend compte d'une limitation dans tous les plans. Le reste de l'examen clinique est normal dont l'examen neurologique ne retrouvant aucun signe radiculaire. Conformément aux recommandations canadiennes, le patient bénéficie d'une radiographie cervicale (Fig. 1A) [1]. Celle-ci retrouve sur l'incidence de profil un fragment osseux en avant du plateau vertébral inférieur de C3. Un scanner (Fig. 1B) confirme l'avulsion $\mathrm{du}$ listel marginal. Le listel marginal (noyau épiphysaire) correspond au débord osseux d'un plateau vertébral en croissance, il sert d'ancrage au disque intervertébral [2]. La maturation complète du listel est lente, elle se termine tardivement (20-25 ans) et cela explique les différences de résistance du plateau vertébral pendant cette période. Les contraintes sur le disque liées au traumatisme vont provoquer cet arrachement apophysaire et montrer une image radiographique de tear-drop like. Il s'agit d'une lésion rare mais bénigne et stable, confirmée à distance par des clichés dynamiques. A contrario (le plus souvent lors d'un traumatisme en flexion-compression), une fracture dite en tear-drop est le témoin indirect d'un rachis cervical instable. La lésion osseuse visible (fracture triangulaire du coin vertébral antéro-inférieur) n'est alors qu'un épiphénomène traduisant une lésion disco-ligamentaire majeure. Le traitement

H. Slimani · P. Kauffmann · P. Le Borgne $(\bowtie)$

Service d'accueil des urgences, CHU de Strasbourg,

hôpital de Hautepierre, 1 avenue Molière,

F-67098 Strasbourg, France

e-mail : pierrick_med@yahoo.fr

P. Le Borgne

Faculté de médecine, Université de Strasbourg,

4 rue Kirschleger, F-67000 Strasbourg

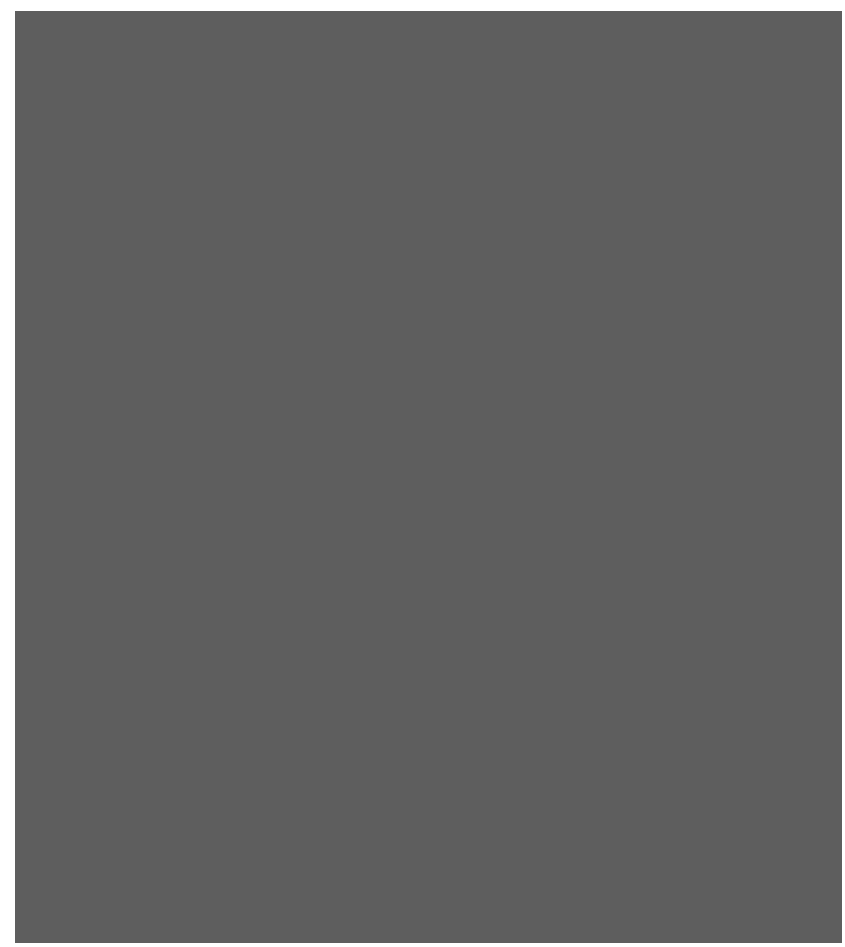

est alors chirurgical (arthrodèse). Le traitement a consisté ici en une immobilisation par collier mousse pendant deux semaines ainsi qu'une antalgie adaptée.

\section{Références}

1. Stiell IG, Wells GA, Vandemheen KL, et al (2001) The Canadian $\mathrm{C}$-spine rule for radiography in alert and stable trauma patients. JAMA 286:1841-8

2. Jonsson K, Niklasson J, Josefsson PO (1991) Avulsion of the cervical spinal ring apophyses: acute and chronic appearance. Skeletal Radiol 20:207-10 